\title{
Subject Index Vol. 189,1994
}

The Subject Index of the 'Proceedings of the Tricontinental Symposium on Autoimmune Skin Diseases' appears in Vol. 189, Suppl. 1,1994 The Subject Index of the 'Clinical and Experimental Dermatology. Festschrift to Professor A. Kint' appears in Vol. 189, Suppl. 2,1994 Acanthoma, clear-cell 437

Acanthosis nigricans 203

Acetone-soluble fraction 58

Acitretin 109,326

Acne therapy 117

Acridine orange 16

Acrodermatitis chronica atro-phicans 430

Acroosteolysis 185

Actinic dermatosis, chronic 241

Acyclovir 332

Aging 193

Agricultural workers 129

AIDS 271,325

Allium 337

Amide group, active 1

Amyloidosis, cutaneous 125

-, systemic 47

Angiodysplasia 292, 392

Angiolymphoid hyperplasia with eosinophilia 87

Annular atrophic lichen planus 95

Anticentriole antibody 23

Anticentromere antibody 23

Antihistamines 117, 146

Antimalarials 425

Antimony 275

Antineutrophil cytoplasmic auto-antibody 69

Antinuclear antibodies 23, 38

Antioxidants 225

Antiphospholipid antibodies 182, 304

- $\quad$ syndrome 421

Arm 90

Arterial embolization 421 Asthma 41, 330

Atopic dermatitis 38, 41, 58, 102, 139,238,331

- $\quad$ - features 41

Atopy 27, 330

Auricular calcification 435 
Barrier creams 320

Bartonellosis 409

Basal cell carcinoma, s. Carcinoma,

basal cell Bath therapy 256 Bazex syndrome 159 Birth cohort 238 Black dots 328 Boomerang phenomenon 415 Borrelia burgdorferi 430 Borreliosis 430 Bowen's disease 78 Brainstem 62

Brown globules 328 Buschke-Ollendorff syndrome 368

Calcification, auricular 435

Calciphylaxis 323,432

Calcipotriol, topical 260

Calcitriol 326

Cancer 271

Carcinogenesis, cutaneous 317

Carcinoma, basal cell 22, 326, 364, 409

-, gastric 207

-, squamouscell 52, 308, 336, 364

-, sweat gland 198

-, verrucous 327

Cardiovascular disease 32

Cataract 41

Catecholamines 350

Child 167

Childhood 133,301

Chlamydia trachomatis 334

Chloroquine 425, 428

Chronic actinic dermatitis 241

- $\quad$ wound 418

Classification 292

Cleansing power 217

Clear-cell acanthoma, multiple,

eruptive 437 Clinical tests 217 Coagulation, blood 32,182 COL7A1 gene 324 Colorimetry 146, 152 Condylomata acuminata 142,396 Confocal microscopy, non-invasive

16 Connective tissue nevus 368 Contact dermatitis 225

-, allergic 332,333

-, irritant 332 Contraception 109,411 Corneosurfametry 152 Cosmetics 55,225 CTCL cell lines 120 Cutaneous amyloidosis 125

necrosis 432

plasmacytosis 251

side effects 402 Cyst, trichilemmal 90 Cytokine 359

treatment 329 Cytomegaíovirus 325

Delayed hypersensitivity 344

Dendrocyte 84

Dennie-Morgan infraorbital fold 41

Dermatitis, cumulative irritant 320

Z > -Dimer 32

Disease concomitance 238

Distress 234 
Down's syndrome 248

Drugreaction 179,196,203,323,

402,412 Dust, house 330 Dyskeratosis congenita 324

Ecthyma 396

Ectopic ossification 435

Eczema 238

Elastic tissue nevus 368

Elastolysis 95, 193, 289

Elastosis serpiginosa perforans 81

Electron microscopy 167,384

Eosinophilic fasciitis 304

- $\quad$ pustular folliculitis 133

Epidemiology 129

Epidermal differentiation, late 336

nevi 170

nevus syndrome 278 Epidermolysis bullosa dystrophica

324

- $\quad$ simplex 173

Epidermolytic keratosis 324

Epithelioid hemangioma 87

Epstein-Barr virus 167

Erythema elevatum diutinum 69

Erythroderma 179

-, bullous congenital ichtyosiform

324 Etretinate 109,410 Eusolex4360 55 Exposure to hypothermia 435

Fasciitis, eosinophilic 304 Factor XI Па 84 Fibrinopeptide A 32

- $\quad$ Bß (15-42) 32

Ficus benjamina 330

Fixed drug eruption 196,412

Flashlamp-pumped dye laser 283

Fluorescence 16

Folliculitis 225 Food intolerance 41

Gastric cancer 207

Genital skin, male 333

Genodermatosis 384

Giant condylomata acuminata 396

congenital nevus 162

porokeratosis 78

Goltz and Gorlin syndrome 323, 327

Granulocyte-macrophage colony-stimulating factor 331

Granuloma pyogenicum 283

Growth factors 418

Hi blockers 146 Haemotherapy, topical 418 Halo dermatitis 269

- $\quad$ naevus 269

Hamartoma 392,437

Handicap 234 
Hay fever 330

Hearing aid dermatitis 333

Heat intolerance 101

Hemangioma 87, 278, 283 Henoch-Schönlein purpura 213 Heparin 33,412 Hereditary benign telangiectasia

286 Heroin 72 Herpes-type virus 167

- $\quad$ zoster 312

Herpes-virus 7325

Hidradenitis, neutrophilic eccrine

211 Histamine 117 HIV 275, 335

- $\quad$ infection 396

HLA 222, 409

Hodgkin's disease 188

Hormone, pure follicle stimulating

321 Human dander 58

papillomavirus 142,308

skin grafts 5 Hydroa vacciniforme 428 Hypergammaglobulinemia 251 Hyperlipidemia 203

Hyperparathyroidism 323, 432 Hyperpigmentation 384 Hypoplasia, focal dermal 323

Idiopathic telangiectasia 286

Ichtyosis bullosa 324

Ig-A 27, 69

Ig-E $27,41,331$

Imaging 16

Immune surveillance 317

Immunity 27

Immunocompetence 52

Immunosuppression 308

Immunotherapy, adjuvant 329

Infants 139

Inflammatory linear verrucous

epidermal nevus 170 Integrins 9 Intercellular adhesion molecule-1 9

- -, soluble 120 Interferon- $\alpha 120,330$

-, recombinant 329

- -2a 321 Interferon- $\gamma 120$ Interleukin-2 120 Interleukin-4 331 Interleukin-6 120,359

Intraosseous angiomas 278 Irritant effect 217 Isotretinoin 62,81,117 Itch 41

Itraconazole 334, 378

Jadassohn-Lewandowsky syndrome 323

Kaposi’s sarcoma 271, 325 Keloid 271,321,322 Keratin 9335 Klippel-Trenaunay-Weber syndrome 292, 392

Langerhans cells 332, 335 Laser 283,286, 322, 327

-Doppler fiowmetry 146 Leg ulcers 322,332,418 Leishmaniasis 275 Leukoderma 162

Leukoplakia, oral hairy 167 Lichen myxedematosus 93

planus 95, 322 Linear dermatoses 170, 278

porokeratosis 78 Linoleicacid 225

Livedo racemosa (reticularis) 421 
- $\quad$ vasculitis 421

Locked-in syndrome 159

Long-term treatment 260

Loratadine 146

Lupus anticoagulant 182,304

- $\quad$ erythematosus 336, 425

Lymph, skin 332

Lymphocyte proliferation assay 323

transformation 344 Lymphoma, cutaneous 325, 326 -, - T-cell 325, 326, 354, 406

Lymphomatoid granulomatosis 9

papulosis 188

Median survival 47 Melanocytic nevus 382

- $\quad$ skin lesions 328

Melanoma 75,120, 162, 265, 382,

318,319,320,326,328 -, metastatic 162, 320, 328, 329 -, ocular 319 Melanosomes 384 Melnick-

Faser syndrome 103 Mental retardation 248 Metastasis 52,207 Metastatic melanoma 162

Meyerson's naevus 269 Microscopy, confocal 16 Mildness 217

Moh's micrographic surgery 326 Moisturizers 320 Molluscum contagiosum 65,396 Monilethrix

410 Monitoring 359 Monoclonal IgA 69 Morphea 99, 157,304,399 Mottled pigmentation 173

Mucin 93, 157 Mucocutaneous leishmaniasis 275

manifestations 47

pigmentation 297 Mycoses 317,334,378 Mycosis fungoides 75, 354, 406 Myxedema 93

Nail 87, 185, 317

Neck 193,289

Necrolytic migratory erythema 72

Neoplasm 271

Neural transmitters 350

Neurofibromatosis 392 Neutrophilic dermatosis 69 Nevus 162,170,265,269,278,

382 -, sebaceous 396

- $\quad$ spilus 265

Nicotinic acid 203

Nile red 16

Nonthiol drugs 1

Nude mouse 5

Nutrition 337

Old age 289 Ofuji's disease 133

- papuloerythroderma 100

Ogilvie's syndrome 312

Oligozoospermia 321

Oncology 318

Onychomycosis 317

Oral hairy leukoplakia 167 Ossification, pinnal 435 Oxybenzone 55

Pachyonychia 323 Paecilomyces lílacinus 320 Palpable mass 406 Palpebral syringoma 248

Pancreas 406 Papular dermatitis 225

- $\quad$ elastorrhexis 368

Papules 289 
Papulosis, hemispherical 159 Parakeratosis pustulosa 413 Parenteral drug abuse 72 Parvovirus B19 213 Pastes 373 Patch test 55,58 Pathogenesis 350 PCR 325, 326, 334, 430 Pemphigus 1,210,337 Penicillamine 81 Perinaevic eczema 269 Petrified ear 435 Pesticides 129 PeutzJeghers syndrome 297 Pharmaceutical creams 65,142 Photoallergy 55

Photochemotherapy 75,234,256 Photodermatosis 428 Photodynamic therapy 321,327 Photopatch test 55 Photosensitivity 241

- $\quad$ disorders 428

Phototesting 241

Phototherapy 313

Pigmentation, mucocutaneous 297

Pityriasis rubra pilaris 313

Plasmacytosis, cutaneous 251

Polyarteritis nodosa 421

Podophyllotoxin 65, 142

Polyclonal immunoglobulins 125

Polymorphous light eruption 241

Polyposis, colonic 215

Porokeratosis 78

Pregnancy 109,411 Prelymphoma 325 Primary biliary cirrhosis 198 Prognosis 162 Prolactinoma 215

Proliferating cell nuclear antigen 354

- $\quad$ trichilemmal cyst 90

Proliferative activity 354

Propionibacterium acnes 117, 344

Propylene glycol 332

Proteus syndrome 392 Prothrombin fragment 32 Pruritus 117 Pseudoxanthoma elasticum 193, 289 Psoriasis 32,234, 238, 256, 260, 297, 340, $359-$, acral 159

PUVA, s. Photochemotherapy Pyoderma gangr(a)enosum 69, 182

Quinacrine 425

Radiopharmaceuticals 318

Recurrence 364

Recurrent pyogenic granuloma 409

Relative risk 238

Renal insufficiency 179,432

Retinoids 62,81,105,109,117,

326,336,410,411 Rhabdomyosarcoma 301 Risk factors 364

Sarcoidosis 198

SAPALDIA study 330

Satellitosis 409

Scleredema 157

Scleroderma, s. Systemic sclerosis

Scraping method 167

Seborrhoeic dermatitis 209

Self-healing cutaneous mucinosis 93

Shampoo 152 
Shower gel 152

Shulman syndrome 304

Siliconegel 322

Sjögren's syndrome 125,317

Skin cancer 207

-, male genital 333

-, non-melanocytic 364

diseases 129

-, induced 416

surface stripping 152

tags, eruptive 215

tests 344 Sneddon's syndrome 421 Soaps 217

Soft tissue hypotrophy 292 Solvents 399 Sonography 146 Spirochetes 430 Spironolactone 402

Spitz's nevus 265 Squamous cell carcinoma,

s. Carcinoma, squamous cell Stem cells 340 Stratum corneum 16, 152

granulosum 16

spinosum 16 Stress, emotional 210 Subcutaneous tumor 392 Sulfaguanidine 196 Sulfonamides 196

Sun protection factor 335

Sunscreening agents 55 Superficial lymphadenopathy 251 Surfactant 152 Sutton's naevus 269

Sweat gland carcinoma 198 Syringoma, eruptive 105 -, palpebral 248 Syringomyelia 185

Systemic amyloidosis 47

- $\quad$ sclerosis 23,99,157, 330, 399

Systemized eruption 170

T cells, activated 332

T lymphocytes 354

Tatoos 322

Telangiectasia in childhood 286

Teratogenicity 109

TGF-receptor 326

Thalidomide 179

Thiol group 337

Thrombin-antithrombin complex

32 Tinea corporis 378

- $\quad$ cruris 378

Tocopherol 225

Tocopheryl linoleate 225

Tomography, whole-body positron

emission 329 Total serum immunoglublins (IgE,

IgA, IgG, IgM) 27 Transit cell 340 Tretinoin, topical 105, 336 Trichilemmal cyst 90

Trichophyton beigelíi 334 Trigger 1,210,337 Tumor necrosis factor- $\alpha$ 120,359 Tumors, immune surveillance 317 -, vascular 318

Ulcers, cutaneous 182, 432 -, leg 322,332,418 Ultrastructure 167,384 Ultraviolet light 241

- $\quad$ radiation 308

Urinary tract infection 139

UVB 313 
Vascular calcinosis 323,432

malformation 292

tumors 87,318 Vehicle 373

effects 373 Videomicroscopy 328 Vimentin 84 Vimentinoma 84 Vitamin E 225

Vitamin-D-resistant rickets 278 Vitiligo 27, 330, 350 Voemer's disease 324

Warts 308, 321 White papules 193 Whitlows, painless 185 Wound healing 418

Xerosis 41

Zovirax 332

444

Subject Index 